Y. Ikeda, C. M. Herr, D. Holzer, S. Kaijima, M. J. Kim. M, A, Schnabel (eds.), Emerging Experience in Past,Present and Future of Digital Architecture, Proceedings of the 20th International Conference of the Association for Computer-Aided Architectural Design Research in Asia CAADRIA 2015, 000-000. (C) 2015, The Association for Computer-Aided Architectural Design Research in Asia (CAADRIA), Hong Kong.

\title{
ASSISTED CONSTRUCTION OF NON-STANDARD WOODEN WALLS AND ENVELOPE STRUCTURES BY PARAMETRIC MODELING
}

\author{
OSCAR GÁMEZ ${ }^{1}$, JEAN-CLAUDE BIGNON² and GILLES \\ DUCHANOIS ${ }^{3}$ \\ 1,2,3 Centre de Recherche en Architecture et Ingénierie ( Map CRAI). \\ School of Architecture of Nancy, France. \\ 19amezboh1@univ-lorraine.fr, ${ }^{2}$ bignon@crai.archi.fr, \\ 3uchanois@crai.archi.fr
}

\begin{abstract}
The non-standard approach, widely discussed in the past years as Architecture progressively uses alternative design methods different from the Euclidean paradigm, has allowed architects to transform the way architecture is conceived and materialized. Such evolution uses computer-aided design along with automation in production to originate the environment for the aided architectural conception field in which we present a method, in its early development stage, intended to create non-standard walls and envelopes based on cellular patterns using wood as base material. We present the results obtained from modeling and building two full-scale prototypes of non-standard wooden walls.
\end{abstract}

Keywords. Non-standard walls; parametric modeling; CNC fabrication; cellular structures; wood construction.

\section{Introduction.}

This paper shows the results obtained through a parametric modeling method whose aim is to create non-standard wooden walls composed by cellular structures as part of the development of a computer-aided architectural design tool. Based on principle of subdividing Nurbs surfaces by cell patterns as an approach to create non-standard facade walls and envelopes, the method applies the concept of mass customization (Anzalone, Vidich and 
Draper, 2008) to structural components since their shape and size properties, when conceived and produced, are dissimilar.

In such context, the non-standard concept applied to wooden-cellular walls characterizes the architectural entity which physical components have different shapes, dimensions and/or materials; escaping from the Fordian paradigm of serial production.

To test the capabilities of our method at its initial development stage two wall prototypes were built as part of the wood challenges, a student contest organized by the School of Architecture of Nancy, France (ENSAN) and the Superior National School of Technologies and Wood Industries (ENSTIB) in the town of Epinal, France from May 13th to 20 2014.

The experiment dealt with two cell morphologies which meant a different approach for modeling, joint management, fabrication and mounting. An interface between the conception and the production environment created a feedback loop that allowed to adapt the parametric model to the production circumstances (delays, machinery type and human capital).

Precedent works in the field of modeling and fabrication of complex wood structures use a 2D fabrication approach for their production. Our method privileges the three-dimensional cutting approach to model and build such structures, so instead of cutting joints perpendicularly to the cutting bed, the machining equipment (CNC milling machines, robots....) have the necessary multi-axis displacement functions and should be able to perform angle-cut joints.

The format exchange from CAD to CAM proved to be complex as it relies on software capabilities to produce fabrication commands without losing information. More specifically, the lack of compatibility between a CAD program and the CAM interface led the parametric model to a series of adjustments that diminished the quality of the produced elements in order to make them feasible by the available production means.

\section{The Non-Standard Concept.}

The non-standard approach, widely treated in academic and professional environments, has emerged as an alternative concept to the Euclidean paradigm and the Fordian serial model. It rests on the principles of shape freedom and assisted production to create non-repetitive structures and architectural objects based on mass-customization (Anzalone, Vidich and Draper, 2008) production systems. 
In this context, the non-standard concept refers to the capacity of creating manifold shaped objects that make part of an architectural or structural object by using contemporary robotic tools.

Precedent referred works analyse design production and integrated digital fabrication (Sass, 2007) and discuss how the CAD and CAM environments are used to achieve the production of architectural objects conceived through digital modeling.

Another approach on Digital Morphogenesis (Kolarevic, 2005) and algorithmic-generated patterns used as a design resource (Artopulos, Roudavski and Penz, n.d.), treat the relationship between cellular biological structures and architecture. They study how modern digital tools allow creating structures that respond to specific architectural needs by employing random biological patterns as a design conceptual basis. Since patterns can take manifold morphologic approaches (Figure 1), they emerge as a mechanism to explore cells as structural components through parametric modeling. We treat these structural cells as "boxes" derived from paneling a Nurbs surface by a cellular pattern (Figure 2).

The cited works refer to CNC fabrication methods, applied to architectural entities, as a part of the conception process. In order to match those techniques a deep knowledge in computing and programming is needed (Scheurer, 2010) for architects, engineers and designers to materialize their work. In that manner, the non-standard approach not only refers to the shape of an architectural entity but to the way it is conceived in function of the means to materialize it.

\section{Method description.}

We present the early development stage of a method used to produce nonstandard facade walls and envelopes, composed by wood-cells (boxes), via parametric modeling and 3D digital fabrication.

Parametric modeling of non-standard walls. A non-standard wall is defined by a Nurbs surface obtained from a specific context which can be a facade outline or a free shape defined by the designer.

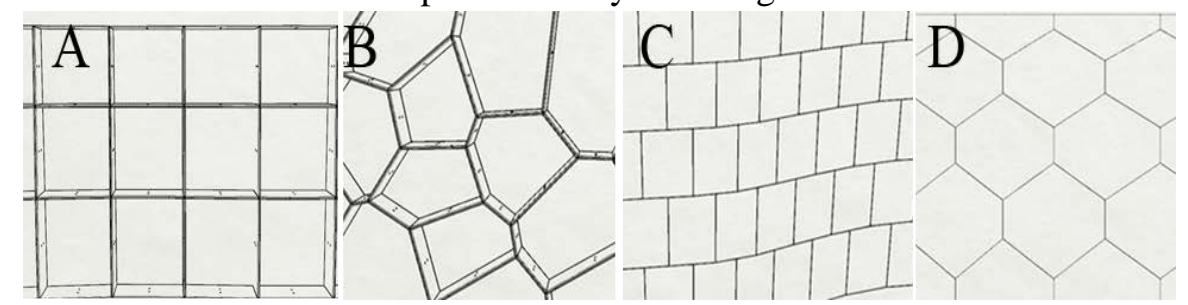

Figure 7. Exploration patterns. A-Quad pattern. B- Voronoi. C-Running bond. D-Hexagonal 
Once the Nurbs surface is modeled, it must be imported into Rhinoceros and treated with the aid of grasshopper following these steps: a) Pattern choice; b) surface subdivision by a pattern; c) creation of cells (boxes) from the panelling process; d) box characterization (cover and cell-wall thickness, joinery) (Figure 2).

The various pattern options, possible to obtain out of parametric tools, offer the possibility to choose between different valid alternatives (Figure 1). Prior to a pattern choice for exploration, an analysis to evaluate the complexity of patterns was performed considering the following aspects:

Pattern density: Since a pattern is derived from a collection of center points placed in a container and the size of each pattern module (cell) is defined by the proximity between these points; the closer the points are, the smaller the cell gets; resulting in an increasing number of structural components and therefore of junction points. Subsequently, the number of elements to deal with, in function of their quantity, is harder to manage (more complex) from the computational point of view (modeling) and the production point of view.

Pattern typology: the complexity concept is bounded to the logical and computational processes needed to render the pattern into a wall structure. Honeycomb patterns and quad patterns are more like regular patterns in which the variables are easier to be controlled from the modeling point of view. But for other patterns like the Voronoi pattern, is different. The Voronoi pattern is more complex since the number of variables to treat cell vertices increases in function of the number of sides of every cell. As a result, the cutting paths are less regular and the cutting path programming is more demanding. Nesting is also different. With a regular pattern, there is more chance to get an optimal use of plywood sheets to avoid waste, however with irregular ones, the amount of waste increases resulting in a more demanding task of material optimization.

This analysis is useful to demonstrate, due to its computational backgrounds, that parametric modeling of cell structures refers its complexity to the concept of disorganized complexity (Weaver, 1948) in which the number of variables of a problem do not follow parallel paths but random paths.

In order to get feedback from pattern complexity and optimize the parametric model, we used which seemed to be the less and the most complex patterns to test: a regular quad-pattern and a Voronoi pattern (Figure 1A \& B). 
All of these aspects are controlled by modeling inputs. Modeling inputs define the parameters to which the parametric model responds to. At the scale of the wall they define pattern morphology, curvature (on $\mathrm{X}, \mathrm{Y}$ and $\mathrm{Z}$ axes), cell creation by paneling (Issa, 2013), cell density, cell size and wall thickness (constant or variable). In the following paragraphs we will refer to cells as boxes.

At the scale of the box, inputs are: a) box-wall and box-cover thickness (inherited from the wood sheet in use); b) joinery (mitres and rabbets); c) cell-connector placement and drilling (bolts) (Figure 4D).

A box is derived from a paneling operation (Figure 2A), the panel boundary defines its creation perimeter, contour walls and covers whose thickness is inherited from the chosen wood sheet. The intersections between these items (box-walls and box-covers) are treated as joints too (Figure 2D).

Every box in a structure is composed by items and those, by more basic elements such as points, faces and edges represented as data in the parametric model (Figure 2B). As the complexity of the structure increases so does data. Through data filtering, the edges of every box are extracted to perform a series of intersections to obtain the points that generate joints like rabbets and mitres (Figure 2D).

Junction points are ordered to create joint profiles and then applied to all of the items in every box (Figure 2C). Some difficulties appeared when doing so, since the point extraction technique does not tolerate excessive wall curvature resulting in erroneous or null joint profiles. For the prototypes presented herein, a positive result was achieved by diminishing curvature on a curved wall; nevertheless, the idea is to obtain curved walls without joint creation being a hard constraint.

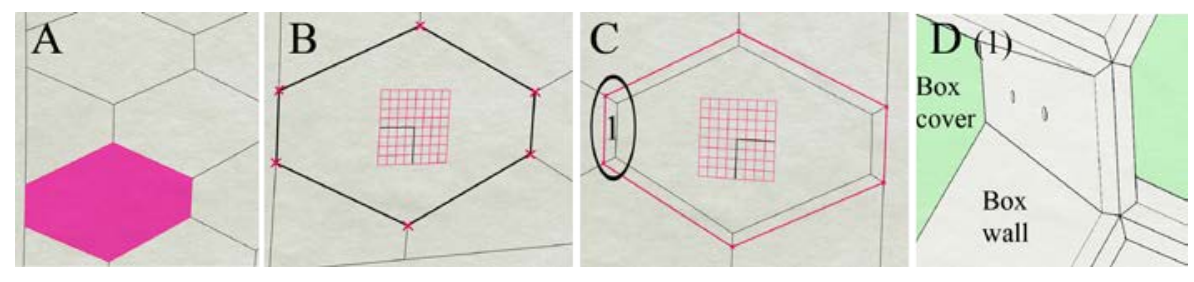

Figure 2. Box generation. A-Surface paneling by a pattern. B-Loop (box) creation by a closed polyline and work plane extraction. $C$ - Box-wall thickness defined by patch subdivision with profile lines. $D$-Box-wall creation by profile extrusion along a plane normal. Mitre joints 


\subsection{Automated fabrication and Production.}

The parametric model allows the numbering, flattening and nesting of structural items previous to the CNC cutting process (Figure 3); however, a manual nesting optimization was made to save material and time during cutting. Prior to the production stage, the following machinery constraints are integrated into the parametric model: a) A CNC Güdel industrial 5-axis robot with an ABB controller; b) Robot tools: a 25mm coarse tooth mill and a 15 ” saw.

These constraints induced changes to the parametric model that match the capabilities of the equipment. For instance, rabbets were forced to a $90^{\circ}$ angle because angle cuts in rabbet operations are not possible to do because of the mill-end diameter. Also, the gap between items after nesting was adjusted to a minimum of $50 \mathrm{~mm}$ so that the mill-end gets room enough to displace without damaging any items when cutting (Figure 3B).

As a result of the interaction of parametric modeling with digital production, a workflow that sets the process to follow when conceiving and fabricating a structure by parametric and robotic means, was defined (A plywood sheet is processed in about 18 minutes by CNC cutting including fastening to the cutting bed, cutting, numbering, clearing and sorting of items. The cutting interface of the robot, simulates the fabrication routine before launching it (Figure 6C), providing information about invalid cutting paths which can be directly adjusted in the CAM interface without repeating the file exchange process (Figure 6B).).

\section{Full-scale prototype production.}

The production stage takes into account two more variables: transportation and mounting. Structural components are prefabricated and their size should be adequate for them to be handled, transported and mounted; such final optimization can be made by increasing cell density (which diminishes box size).

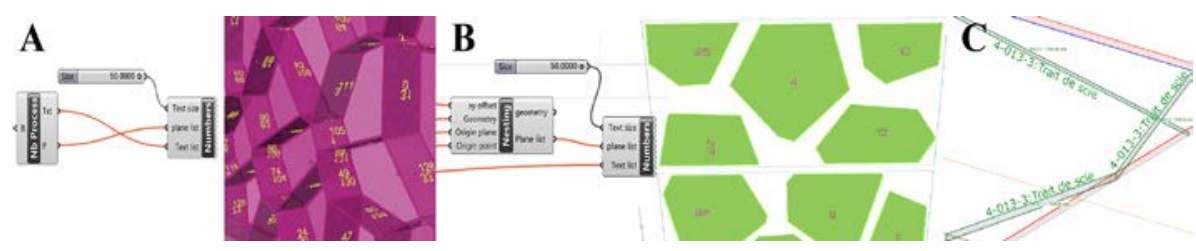

Figure 3. A-On-model tagging. B-Nesting. C-Cadwork BTL instructions. 
For our prototypes, handling was not the only premise before producing the structures though a schedule optimization was critical. A projection established that the production stage would take more time than available, so a balance between handling and production efficiency had to be found.

The optimization of box density in the structures improved the production schedule in 48 working hours by reducing the grid structure from 60 to 24 boxes and the Voronoi structure from 38 to 18 boxes (Figure 4).

A file exchange is also necessary to obtain the CAM commands that lead to CNC cutting. To do so, a "Step" file exported from Rhinoceros is imported into Cadwork to produce the BTL files (Cadwork and SEMA, 2014) that the CAM interface (LignoCAM) uses to communicate with the robot controller (Figure 3C \& Figure 6A-B). Some difficulties emerged since the Cadwork interface did not read some milling operations like acute angles and angled mitres from the imported geometry. This led to manual programming of several BTL instructions.

A plywood sheet is processed in about 18 minutes by CNC cutting including fastening to the cutting bed, cutting, numbering, clearing and sorting of items. The cutting interface of the robot, simulates the fabrication routine before launching it (Figure 6C), providing information about invalid cutting paths which can be directly adjusted in the CAM interface without repeating the file exchange process (Figure 6B).

Despite the fact of using parametric modeling as main tool, the method is not fail-safe yet. For the Voronoi structure, a complication with the mill-end occurred when some items of the Voronoi structure could not be fabricated due to high acute angles in mitres so that manual production or finishing was necessary (Figure 7B\&C). After cutting and classifying the items, the boxes were assembled and a mounting test was executed before sending the structural components to the construction site. It took two days to mount the finished prototypes (Figure 8).

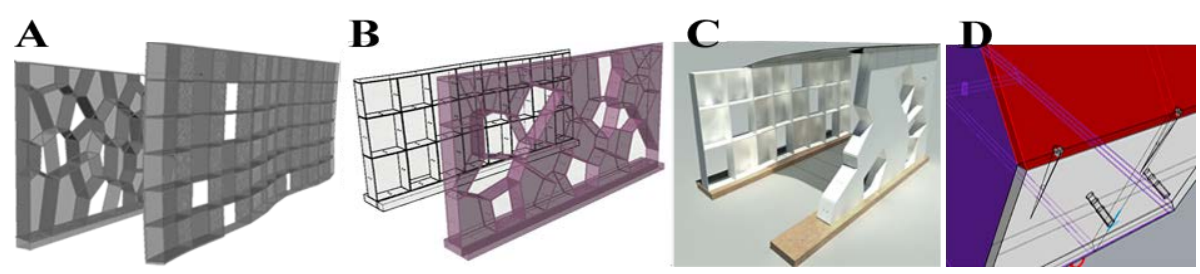

Figure 4. Box density optimization (quad/voronoi) $A, 60 / 38 \cdot B, 24 / 30 \cdot C$, 24/78.D, Assembly detail on mitre joints. Items are put together by using fasteners and bolts. 


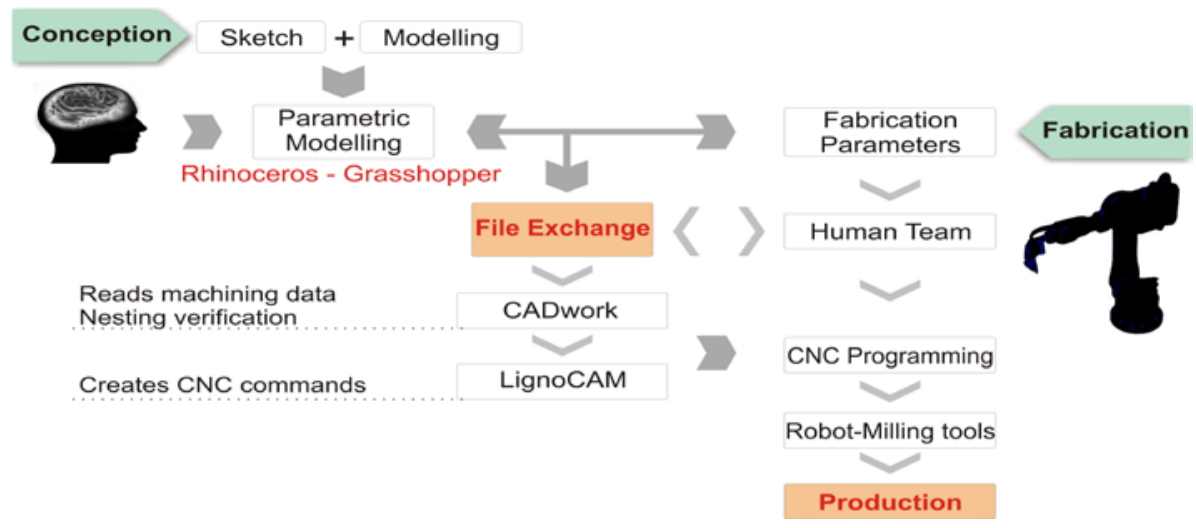

Figure 5. Conception to production workflow

\section{Discussion.}

We presented a method that deals with the conception by parametric modeling of non-standard wooden walls, suitable for being used in facade rehabilitation projects or as a design tool for new buildings whereby they can get to perform as energetically passive architectural envelopes.

By building two full-scale prototypes we found a series of problems that need to be avoided for this method (and others similar to it) to succeed since parametric modeling manages the necessary data to model and fabricate joints such as mitres and rabbets repeating them over different shaped structural elements regardless of the pattern variations and geometric deformations of the structure of which they make part.

A first improvement should be stop using third-party software to create de CAM routines exported from Rhinoceros to LignoCAM, the loss of information generated with the file exchange proved to be significant and led to several mistakes when reprogramming the prototypes' cutting routine.
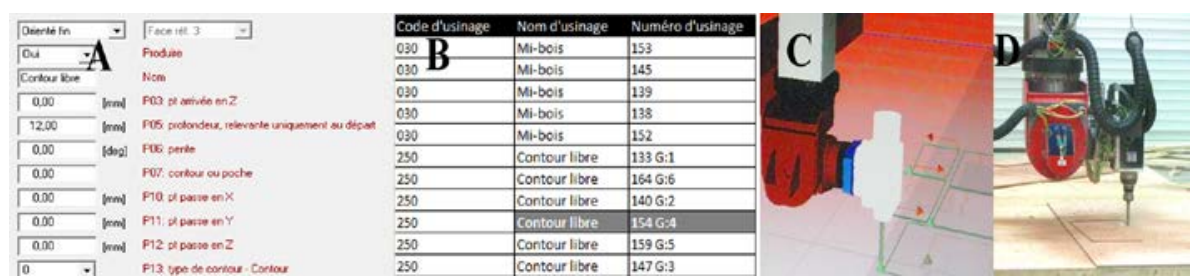

Figure 6. A, CAM commands. B, ISO cutting path table. C, Cutting path simulation.

D, CNC cutting. 

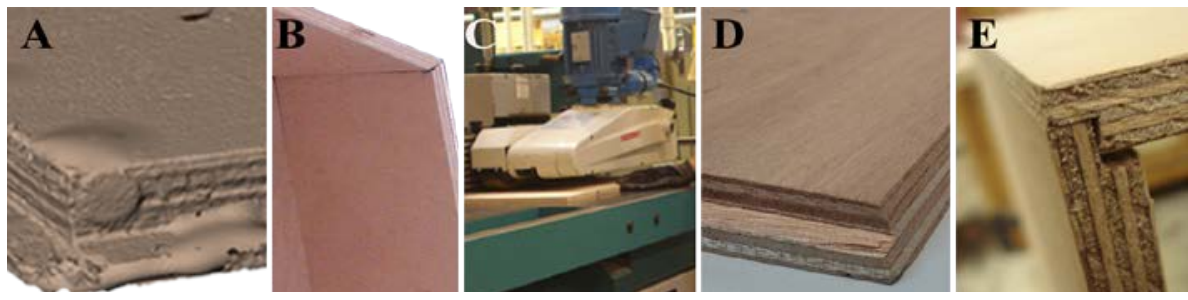

Figure 7. A, 3d scanned image of an unsuccessful rabbet. B, Mitre joint. Angles not coupling well after cutting. $C$, Manual correction of mitres and rabbets. $D$, An item containing both a successful mitre and rabbet. $E$, Correct coupling with rabbet joints.

In no way the production of these walls should be limited by the capabilities of a machine, so the right set of tools should be available to produce accurate wood-detail operations. In such case, a mill-end of about $8 \mathrm{~mm}$ diameter should be used; it would allow performing acute and straight cuts without major restrictions. As time is important too, the human team in charge of automated machinery should be familiar with such operations in order to avoid unnecessary tests and mistakes.

\section{Conclusion.}

Having tested two joint types, it becomes clear that joinery treatment is a complex task to be performed from the modeling and production point of view. The success of any cellular structure intended for non-standard walls, as it is the case, depends heavily on the joinery conception method, a process that must take into account the production means available for such task.

The results showed herein, regarding the modeling and construction of two cell-patterned wall prototypes as an early research stage on this matter, proved the feasibility of such structures by using basic joint types which leads to think that a more complex approach is possible in order to improve the method, take it to a more practical level and apply it on actual buildings as a facade rehabilitation alternative, which is the next stage of research.

\section{Acknowledgements.}

Special thanks for the technologic and human support from the ENSTIB, the Laboratory for Studies and Research in Wood (LERMAB) in Epinal France and the School of Architecture of Nancy. 

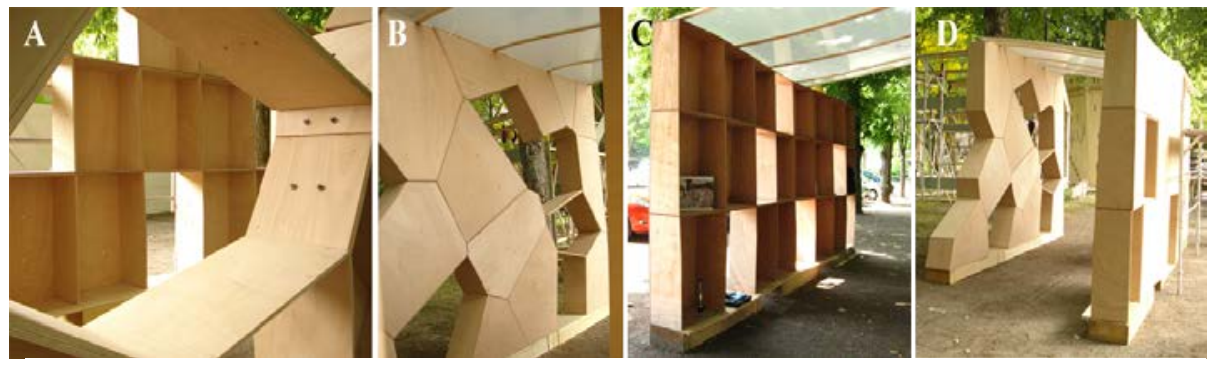

Figure 8. Finished wall prototypes. A, Grid-pattern wall view through a voronoi cell. B, Voronoi-patterned wall. C, Grid-pattern finished wall. D, Finished prototypes.

\section{References}

Anzalone, P., Vidich, J. and Draper, J. (2008) Non-Uniform Assemblage: Mass Customization in Digital Fabrication, In Without a Hitch: New Directions in Prefabrication Architecture |, UmassAmherst, [online] Available at: http://scholarworks.umass.edu/wood/2008/ (Accessed 18 November 2014).

Artopulos, G., Roudavski and Penz, F. (n.d.) Adaptive Generative Patterns, In Proceedings of The Second International conference of the Arab Society for Computer Aided Architectural Design, Sharjah: the Arab Society for Computer Aided Architectural Design (ASCAAD), 2006, Jamal Al-Qawasmi and Zaki mallasi, pp. 341-362.

Cadwork and SEMA (2014) design2machine - the data transfer interface for wood constructions - btl v10, the data transfer interface for wood constructions - btl v10:, [online] Available at: http://www.design2machine.com/btl/index.html (Accessed 8 July 2014).

Issa, R. (2013) PanelingTools for grasshopper, Robert McNeel \& Associates, [online] Available at: http://v5.rhino3d.com/page/panelingtools-downloads (Accessed 10 March 2014).

Klein, F. (1893) A comparative review of recent researches in geometry, Bulletin of the American Mathematical Society, 2(10), pp. 215-249.

Kolarevic, B. (2005) Architecture in the Digital Age: Design and Manufacturing, New Ed edition, New York, Taylor \& Francis.

Sass, L. (2007) Synthesis of design production with integrated digital fabrication, Automation in Construction, 16(3), pp. 298-310.

Scheurer, F. (2010) Materializing complexity, Architectural Design, 80(4), pp. 86-93.

Sevtsuk, A. and Kalvo, R. (2014) A freeform Surface Fabrication Method with 2d Cutting, In 2014 Proceedings of the Symposium on Simulation for Architecture and Urban Design, Tampa, Florida, Dr. David Gerber and Rhys Goldstein, pp. 109-116, [online] Available at: http://www.simaud.org/proceedings/.

Weaver, W. (1948) Science and complexity, American Scientist, 36(4), pp. 536-544. 